\title{
The perception of rotated shapes: A process analysis of shape constancy*
}

\author{
DOMINIC W. MASSARO $\dagger$ \\ University of Wisconsin, Madison, Wisconsin 53706
}

\begin{abstract}
Ss made objective shape judgments of circular objects rotated in depth to provide a process analysis of shape constancy. The significant finding was that task difficulty, as reflected by proportion errors and correct reaction times, increased with increases in rotation from the frontal-parallel plane. This effect was located at the perceptual encoding stage of the shape judgment process. It was demonstrated that, in contrast to true shape information, the time to interpret slant and two-dimensional projected shape information was not critically dependent on degree of rotation. These results and a number of other additional observations demonstrate that the invariance hypothesis does not provide a sufficient account of shape constancy. Although projected shape and slant judgments can be made easily, perception of true shape involves encoding a figure-ground relationship by a process that does not rely exclusively on the discrete values of projected shape and slant.
\end{abstract}

Perceived shape usually corresponds to objective shape. The principal account of this observation is the shape-slant invariance hypothesis, which states that perceived shape is uniquely determined by projected shape and apparent slant (Koffka, 1935; Beck \& Gibson, 1955). The projected shape is available in the retinal image, and apparent slant is determined by depth cues. Two experimental findings have been offered as support for the invariance hypothesis. First, the predicted relationship between perceived shape and apparent slant has been substantiated (Kaiser, 1967; Winnick \& Rogoff, 1965). Second, a number of studies of shape perception have shown that shape constancy breaks down when cues to orientation are reduced or eliminated entirely (Epstein \& Park, 1963; Winnick \& Rosen, 1966). Although it has not been done, it could be shown that reliable shape perception also fails when cues to the projected shape are diminished. In both cases, it is not surprising that removal of stimulus information can decrease veridical perception.

The invariance hypothesis supplies a simple algorithm for shape perception using the laws of projective geometry. The computational algorithm is essentially a geometrical equation that provides the true shape of an object given the retinal image shape and accurate registration of the rotation of the figure. Application of the invariance hypothesis is not confined to the case of accurate registration of slant. If slant is over- or underestimated, the perceived slant would be inserted in the algorithm and a unique shape would be perceived,

\footnotetext{
*This research was supported, in part, by a research grant from the Wisconsin Alumni Research Foundation. The author thanks Sheldon M. Ebenholtz, William Epstein, and Wayne Shebilske for providing a staunch and consistent defense of the invariance hypothesis.

$\dagger$ Requests for reprints should be sent to Dominic W. Massaro. Department of Psychology, University of Wisconsin, Madison, Wisconsin 53706 .
}

although the perceived shape would be nonveridical. It would also be reasonable to allow projective shape to be registered inaccurately, providing a subjective estimate for projective shape. However, it is important to note that the algorithm of the invariance hypothesis does not change, even though subjective estimates of slant and projected shape are used.

The first purpose of this research is to test the invariance hypothesis account of shape perception. The second objective of the present experiments is to contribute an experimental approach to the analysis of the process underlying the achievement of shape constancy. In this approach, the shape-judgment task is assumed to involve a sequence of mental processes (stages) between presentation of the stimulus and S's discriminatory response. Consider a simplified version of a procedure for assessing shape constancy. On each trial, an ellipse or a circle test figure is presented at one of a number of various orientations. The task for $S$ is to report whether the test figure is the ellipse or the circle.

Figure 1 shows a simple stage model of this task. The $S$ can be conceptualized as first determining the shape of the figure in the perceptual encoding stage. The invariance hypothesis has been proposed to account for this stage of processing. However, two other processes are involved in the task. After encoding the shape, some comparison must be made with the possible alternatives in the task. The $S$ then selects the response that agrees with the outcome of the comparison process. The simplest assumption is that each of these stages is independent and sequential, à indicated in Fig. 1. Each stage takes some finite time and outputs information to the following stage. The time to complete a stage is independent of the time it takes to complete preceding or following stages.

In terms of the model. the present experiments ask whether the invariance hypothesis can account for the time it takes to carry out the perceptual encoding stage. 


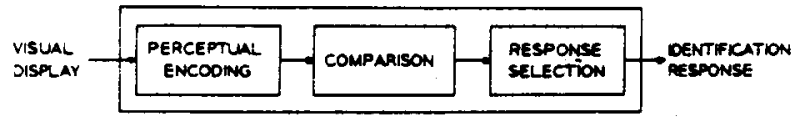

Fig. 1. An information processing model of the shape judgment task. The time to complete each state of processing is independent of the time taken by preceding or following stages.

The findings of Experiment I establish the focal point of the investigation. Experiment I was suggested, in part, by a finding that has been reported by a number of investigators (Smith, 1956; Leibowitz, Mitchell, \& Angrist, 1954), concerning the relationship between the degree of shape constancy and the degree of rotation from the frontal-parallel plane (FPP). Shape constancy declines as the degree of rotation increases. If it may be assumed for the experiments in question that slant (rotation) and projected image were registered accurately at all degrees of rotation, then the decreasing constancy function presents a serious difficulty for the invariance hypothesis. Given that the invariance algorithm is always consistent, task difficulty should not increase with increases in degree of rotation.

In terms of the present stage model, this result could imply that the time to complete the perceptual encoding stage increased with increases in the degree of rotation. Accordingly, when $\mathbf{S}$ is encouraged or is inclined spontaneously to respond in the same time for the target at each degree of rotation, errors will increase with increasing rotation due to incomplete processing. Experiment I examined this interpretation by securing reaction times (RTs) for judgments of rotated shapes (a) when $\mathrm{S}$ was instructed to respond rapidly with a maximum fixed latency (speed instructions) and (b) when $S$ was instructed to respond errorlessly (accuracy instructions). The speed instructions should yield increasing frequencies of errors for increasing degrees of rotation accompanied by a relatively constant RT at all rotations. The accuracy instructions should yield increasing RTs for increasing degrees of rotation accompanied by relatively accurate shape judgments at all rotations.

\section{EXPERIMENT I}

\section{Method}

\section{Apparatus}

The apparatus consisted of three units: a display box, a response panel, and a timing control unit with a clock counter. The display box was $45.7 \mathrm{~cm}$ across the front, $76 \mathrm{~cm}$ long, and $61 \mathrm{~cm}$ high. The box was mounted on 17.8- $\mathrm{cm}$ legs and was located on a table. The figures were mounted on vertical rods, $3 \mathrm{~mm}$ thick. Five centimeters of the rods were visible. The stands to the figures were occluded by a board painted black and placed $44.5 \mathrm{~cm}$ from the front of the box. The figures were presented $73 \mathrm{~cm}$ from S's eyes and at eye level. The gray figures were $8 \mathrm{~cm}$ from the black background of the display. The figures were illuminated by two $28-\mathrm{cm}$ fluorescent lights, mounted horizontally $17.8 \mathrm{~cm}$ below and $17.8 \mathrm{~cm}$ above eye level on the inside of the front end piece. The interior of the box was painted black. The Ss viewed the figures binocularly through a window $(11 \times 4 \mathrm{~cm})$ with an extended viewer. The extended viewer limited any large head movements. The window was located $5 \mathrm{~cm}$ from S's line of sight. A rotating shutter, placed $4 \mathrm{~cm}$ behind the window, was used to occlude S's view of the figures. The shutter took approximately $1 \mathrm{sec}$ to raise completely; however, because of the small window, the complete figure appeared as a whole.

The response panel was a box $(28 \times 21.5 \times 4 \mathrm{~cm})$ with two pushbuttons, each mounted $6.3 \mathrm{~cm}$ from the side and front, respectively. The response panel was located under the front end of the display box, and $S$ was free to adjust the response panel so that he could hold his two index fingers on the two pushbuttons while looking into the display box. The timing unit consisted of a reset button, which opened the shutter and started the clock, operating at .001-sec intervals, and indicator lights to tell $E$ which response was made. When a response was made, the clock stopped, the shutter clused, and a light indicated to $\mathrm{E}$ what response was macie.

\section{Stimuli}

The practice stimuli used to familiarize $S$ with the pushbutton RT task were two blue and two green rectangles, all the same size. The $S$ responded to these practice stimuli on the basis of color (blue or green). The two test stimuli were flat two-dimensional figures, a circle with a diameter of $5.1 \mathrm{~cm}$ and an ellipse with a vertical axis of $5.1 \mathrm{~cm}$ and a horizontal axis of $4.3 \mathrm{~cm}$. The figures were $3 \mathrm{~mm}$ thick and were presented at a distance of $73 \mathrm{~cm}$ from S. Accordingly, the horizontal axes of the circle and elipse subtended visual angles of 4 and $3.4 \mathrm{deg}$, respectively, when presented in the FPP. The luminance of the figures was $4.62 \mathrm{fL}$. Given that the figures were viewed binocularly, convergence and binocular disparity were available as indicators of slant. However, since the figures were homogeneous in color and the lighting was uniform, most of the standard monocular cues of relative depth and slant were eliminated.

\section{Subjects}

Ten students were obtained by advertising and were paid $\$ 6$ for participation in the experiment.

\section{Procedure}

Each $\mathrm{S}$ was tested on 5 successive days. On the first day, the instructions were read to $S$, and he was shown the circle and ellipse and their positions in the box. The instructions for all the shape-judgment experiments stressed objective shape matching rather than phenomenal or apparent shape matching.

"When you look into this box, you will see a single figure. I am interested in how fast you can judge the physical shape of the figure. If the figure is a circle, push the right (left) button with your right (left) index finger. If the figure is an ellipse, push the left (right) button with your left (right) index finger. This means that after you have made a judgment of 'circle,' I should be able to take the figure, lay it over a circle, and find that it corresponds perfectly in all dimensions. Similarly, a figure you judge to be an ellipse should exactly overlay an ellipse. Please remember that you are to judge the actual physical dimensions of the figure. To make this clear, suppose you are looking at a dinner plate at eye level. The plate at eye level may look very elliptical, but if you were asked to judge its shape you would probably say circle. This is what I wish you to do here. Judge the actual physical shape of the figure.

"The figure will be either a circle or an ellipse. The figures will be either flat or rotated about a vertical axis (demonstrate). A 
circle may be rotated so that it looks to have the same shape as a flat ellipse (demonstrate). In this case, you would respond 'circle,' since the actual physical shape of the figure is circular. The figure will remain visible until you press a button. On half of the trials, the figure will have a circular shape, and on half, the figure will have an elliptical shape. The figures will be presented in a perfectly random order. Don't try to anticipate the next trial."

The instructions for five of the $S$ s encouraged $S$ to respond as rapidly as possible compatible with reasonable standards of accuracy. It was implied that a reasonable number of errors would be accepted, but that slow responding was undesirable. In fact, when the RT exceeded $1.4 \mathrm{sec}, \mathrm{S}$ was prompted to respond more rapidly on the succeeding trials. The instructions for the other five Ss emphasized the importance of accuracy: "Press the appropriate button as fast as you can without making an error." The S was told that we were interested in "how fast you can make the correct judgment." These Ss were given feedback on error trials, and all trials on which an error was made were repeated sometime later in the series.

The task in Experiment I required Ss to identify the physical shape of single figures (circle or ellipse) presented either frontal parallel or at one of six rotations $(13,26,39,52,65$, or $78 \mathrm{deg}$ ) about the vertical axis. In each instruction condition, two Ss responded to the circle with their dominant hands and three Ss responded to the ellipse with their dominant hands. The combinations of 2 figures by 7 rotations were randomized within blocks of 14 trials for 5 blocks. Thus, on any one day, a S received 70 experimental trials. A different set of random blocks was used for each $\mathrm{S}$ for each day. The approximate average intertrial interval was $10 \mathrm{sec}$, and the figure was always removed and replaced. whether a change was called for by the random sequence or not. The first day was considered practice and the data disregarded. The following 4 days were identical to the first day, except that the instructions were not read and the experimental targets were not shown to $S$ prior to the first trial. Twenty-four trials of colored rectangles (blue or green) were given as practice on Day 1 , and 12 trials were given on the following days before the experimental task.

\section{Results and Discussion}

Figure 2 shows the mean percentage errors and the RTs of both correct and incorrect judgments as a
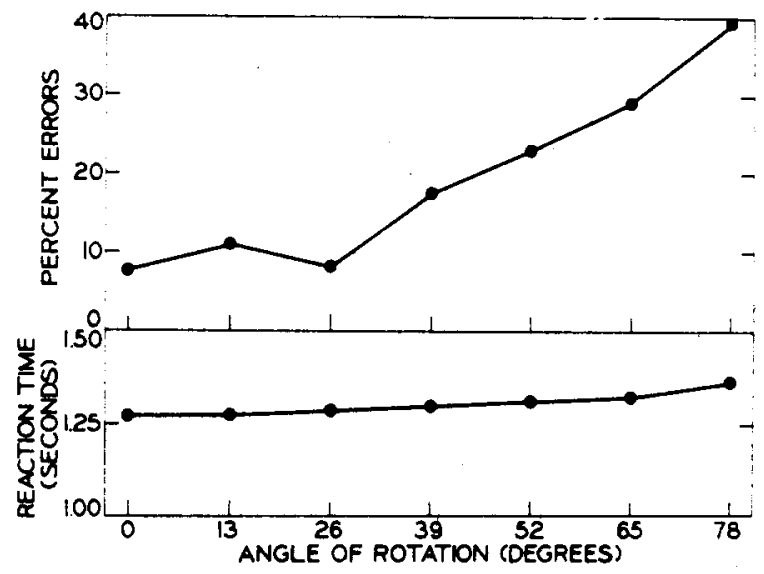

Fig. 2. Mean percentage errors and mean RTs of correct and incorrect judgments in Experiment $I$ as a function of angle of rotation of the test figure. (The Ss were tested under speed instructions.)

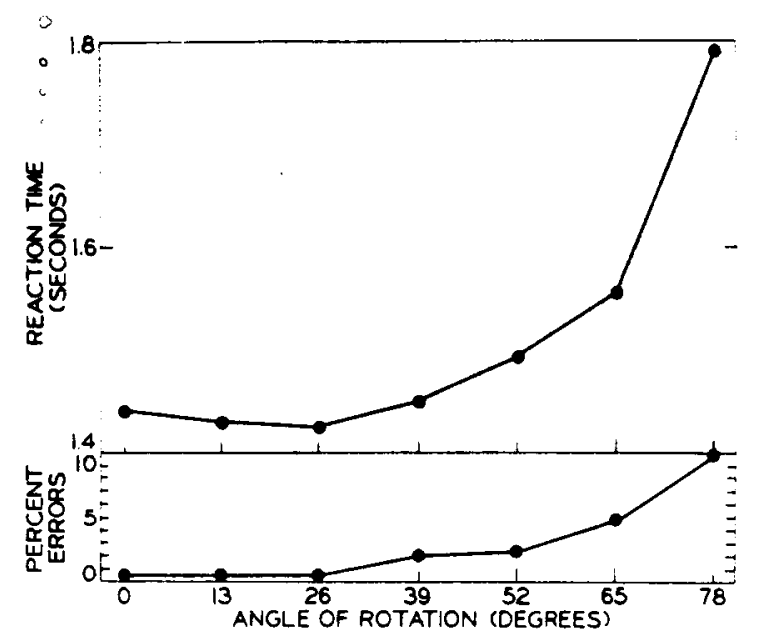

Fig. 3. Mean RTs of correct judgments and mean percentage errors in Experiment $I$ as a function of angle of rotation of the test figure. (The Ss were tested under accuracy instructions.)

function of angle of rotation for the Ss given speed instructions. As can be seen, the bottom panel of Fig. 2 shows that the speed instructions were successful, since RTs were relatively constant across angle rotation. Accordingly, percentage errors should reflect task difficulty if increasing task difficulty increases necessary processing time. The top panel of Fig. 2 shows that percentage errors increased sharply with increases in angle of rotation beyond $26 \mathrm{deg}$. For each of the five Ss, errors increased with increases in angle of rotation.

The error data are consistent with the earlier reports that constancy (veridical shape judgment) declines as degree of rotation increases. According to the present analysis, this trend is due to the restrictions imposed by the procedure on processing time. Figure 3 presents the results for the Ss given accuracy instructions who, therefore, could adapt their reaction times to the demands of the task. The bottom panel of Fig. 3 shows the mean percentage errors as a function of angle of rotation. As expected, the errors were sharply reduced with accuracy instructions, although errors continue to increase sharply with increases in degree of rotation. The RTs for correct responses in Fig. 3 show what the $S$ has done to achieve better accuracy. The top panel of Fig. 3 shows that angle of rotation had a very substantial effect on RT. For all Ss, increasing angle of rotation beyond 26 deg increased RTs in a positively accelerated manner. Thus, as the task became more difficult, Ss utilized more processing time to secure the desired outcome. Confirming this conclusion is the impressive correspondence between the shape of the RT function in Fig. 3 and the percentage error function in Fig. 2. Both functions are flat to $26 \mathrm{deg}$ and increase with further increases in angle of rotation.

The principal finding of Experiment I has been that shape judgments become more difficult with increases in degree of rotation. Therefore. the time necessary for 
processing also increases with degree of rotation. The experiments that follow were aimed at identifying the stage of processing that is involved. It is possible that perceptual encoding time and comparison time could increase the increases in degree of rotation. For example, the results of Experiment I may reflect the fact that the projected shapes of the figures become more similar with increases in rotation. This possibility is tested in Experiment V; however, first it is necessary to locate the actual stage of processing that is responsible for the results.

It is unlikely that the increase in RT across rotation reflects increases in the response selection stage. According to the stage model, changes in degree of rotation affect the stimulus information available to the perceptual encoding stage. Logically, there is no reason why rotation should affect response selection, since it is assumed that the shape judgment is completed before response selection begins. Accordingly, the time for response selection should be constant and independent of the time taken by the earlier processing stages. A number of previous studies have reported results that indicate response selection time is not dependent on the time to complete earlier stages of the RT process (Sternberg, 1969). In the shape judgment task, it is unlikely that increases in rotation significantly affect the time for response selection.

The comparison stage might account for the increase in reaction times with increasing orientation. For example, it is possible that $S$ could hold a visual image of the figures as they are seen in the frontal-parallel plane. Accordingly, if the figure is presented in the frontal-parallel plane, matching time would be minimal. Increasing the rotation of the figure would slow down the matching process, since the $\mathrm{S}$ would have to operate on the shape of figure presented or the representation of the figures in memory to align their orientations. A mental operation of this sort, a rotation in imagination, was alluded to by Helmholtz in 1867 (Warren \& Warren, 1968, p. 128) and more recently by Shepard and Metzler (1971).

Shepard and Metzler (1971) studied the comparison of perspective line drawings of three-dimensional objects. Each object was built with solid cubes attached face-to-face, forming an arm-like structure with right-angle elbows. The Ss viewed two different perspective projections and responded as to whether the objects were the same or different in true shape. The independent variable was the difference in orientation along the vertical axis between two perspective views of the same shape. The different trials contained one of the original figures of a same trial, but paired with the other figure reflected about some plane in three-dimensional space.

The RT was recorded as a function of differences in orientation for the same pairs. The results indicated that same RT was a linear function of the angular difference between two projections of the same three-dimensional object. Shepard and Metzler interpreted this result as reflecting a process of "mental rotation in three-dimensional space [p. 703]." The slope of the "same" RTs indicated that the Ss were able to rotate the objects at about $60 \mathrm{deg} / \mathrm{sec}$. The authors also suggested that most of the reaction time could be accounted for by "mental rotation" rather than by a preliminary process of preparation or search (perceptual encoding in the present model). Certainly, the linear increase in RT across rotation differences must be assumed to represent only "mental rotation" for the rotation hypothesis to be internally consistent. Another process could consume more time for increases in rotation differences, but there is no reason that this process should be linear.

In contrast to Shepard and Metzler's conclusion, it seems that the figures must have undergone a good deal of processing other than mental rotation. The linear orderliness and small variance in the "same" reaction time indicate that the Ss almost always chose the shortest direction of rotation. Without any preprocessing, Ss should have rotated in the direction greater than $180 \mathrm{deg}$ on half of the trials if the direction of rotation was chosen randomly. If this was the case, RTs would not have been directly related to rotation differences.

The "mental rotation" operation of comparison might account for increases in RT with increases in angle of rotation in the present experiment. We could assume that $\mathrm{S}$ maintains a mental representation of how the circle and ellipse looked in the FPP. Presented with a figure oriented in depth, $\mathrm{S}$ rotates that figure to the FPP and compares it to the mental representations in the FPP. And, if rotation time increases with increases in angle of rotation, it would be possible to obtain the results that were observed in Experiment I. However, RT was not a linear function of rotation. If $S s$ were performing a rotation into the FPP, it was somehow more difficult to go, for example, from 78 to $65 \mathrm{deg}$ than from 13 to 0 deg.

It is, therefore, necessary to provide a test of the rotation hypothesis of the comparison stage in the experimental paradigm with circles and ellipses. To do this, the absolute judgment task was changed to a same-different comparison task with two figures. The rotation hypothesis says that the critical variable in a same-different task is the difference in rotation between the two figures. According to the rotation hypothesis, the $S$ is assumed to rotate one figure into another to determine if they are the same or different in shape. An experiment was conducted which held the difference in rotation constant by presenting both figures at the same orientation while varying the absolute orientation of the figures. The rotation theory of the comparison process predicts that RT should not differ across the conditions of absolute rotation because no rotation is required to determine whether the figures are the same or different when the figures are presented in the same orientation. Therefore, RT should be constant across levels of 
absolute rotation if the rotation operation is critical in the present shape judgment task.

\section{EXPERIMENT II}

\section{Method}

\section{Subjects}

Ten Ss were used. Some were obtained through advertising and were paid $\$ 10$. The other Ss were undergraduates who received credit points for an introductory psychology course.

\section{Stimuli}

The targets were the same as those used in Experiment I. The two targets were separated by $17 \mathrm{~cm}$ center-to-center.

\section{Procedure}

The Ss were tested on successive days for five sessions. Prior to the experimental task on each day, Ss were practiced in a same-different RT task, using colored rectangles (blue, green) presented in the frontal plane. Twelve practice trials were given. Fifty-six experimental trials were given per day. If an error was made, the trial was repeated towards the end of the session. The four figure pairs (circle/circle, circle/ellipse, ellipse/ellipse. ellipse/circle) were presented twice at each level of rotation $(0$, $13,26,39.52,65$, and $78 \mathrm{deg}$ ) about the vertical axis. The 56 trials were presented in a different random order per day. The Ss performed the same-different task under accuracy instructions, and objective shape-matching was stressed. For five of the Ss, the left figure was rotated counterclockwise and the right figure was rotated clockwise. For the other five Ss, both figures were rotated in the counterclockwise direction so that the figures were presented in parallel planes. These five Ss were informed that the two forms on each trial would always be presented in the same orientation.

\section{Results and Discussion}

Before reporting the significant findings, two results

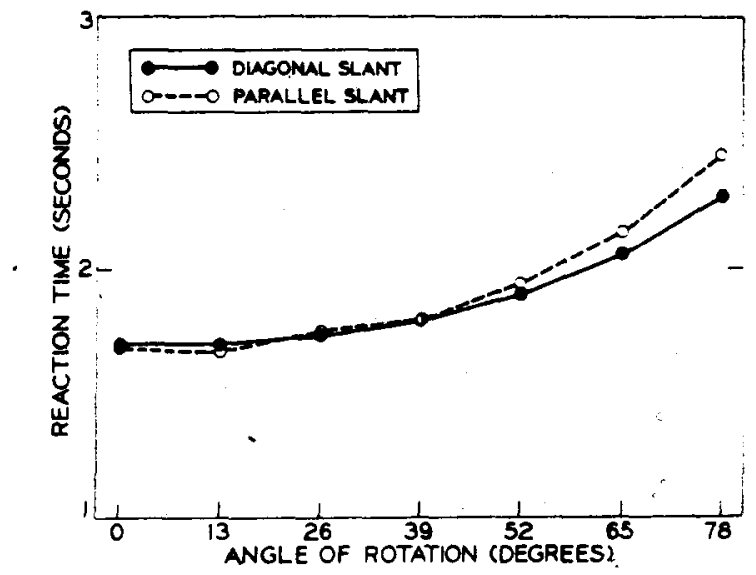

Fig. 4. Mean RTs of correct judgments in Experiment II as a function of the angle of rotation of both figures. (The variable diagonal or parallel slant designates whether the figures were presented in diagonal or parallel planes.)
Table 1

Mean Percentage Errors in Experiment II as a Function of the Angle of Rotation of Both Figures*

\begin{tabular}{lrrrrrrr}
\hline & \multicolumn{6}{c}{ Angles of } & \multicolumn{7}{c}{ Rotation (Degrees) } \\
\cline { 2 - 8 } \multicolumn{1}{c}{ Slant } & 0 & 13 & 26 & 39 & 52 & 65 & 78 \\
\hline Diagonal & 1.2 & 1.2 & .6 & 1.2 & 3.0 & 5.3 & 16.7 \\
Parallel & 0.0 & 3.0 & 1.2 & 5.3 & 9.6 & 17.0 & 32.2 \\
\hline
\end{tabular}

*The variable diagonal or parallel slant designates whether the figures were presented in diagonal or parallel planes.

should be reported. First, the RTs for same and different responses did not differ significantly from each other at any of the rotation conditions. Second, as expected, the variances of the RT scores were positively correlated with their means. This positive correlation was also found for the error scores. These two results were also found in each of the following experiments and will not be discussed further.

The results shown in Fig. 4 and Table 1 are straightforward. Mean percentage errors and mean RT increased with increases in absolute angle of rotation in a positively accelerated manner under both rotation conditions. When the figures were presented in parallel planes, comparison should not have required any mental rotation. The results disconfirm the rotation hypothesis as a sufficient condition for the RT functions in this task. Since the two figures were always equal in angle of rotation, no difference in RT should be expected if rotation was a sufficient condition for increases in RT. The similarity between the two curves in Fig. 4 also shows that the critical variable is degree of rotation from the FPP, not rotation differences. Therefore. the mechanism responsible for increasing $\mathrm{RT}$ with increases in rotation in our experiments cannot be due to "mental rotation."

Since the RT increase is not due to extra processing at the response selection stage or the comparison stage. the increase must be due to extra processing at the perceptual encoding stage (cf. Fig. 1). The next three experiments provide a test of whether the invariance hypothesis can account for the RT increase with increases in absolute rotation.

According to the shape-slant invariance hypothesis, perceptual encoding consists of an integration of the projected image shape with the perceived orientation of the figure. Increases in RT with increases in absolute rotation could result from the determination of either or both of these variables. i.e., registration of projective shape and/or slant. Experiment III asks for true shape judgments, Experiment IV asks for slant judgments. and Experiment $V$ determines the time it takes for two-dimensional projected shape judgments. If the invariance hypothesis is correct. the perceptual encoding time for true shape judgments should be a composite of the encoding times for slant and projective shape judgments, respectively. 


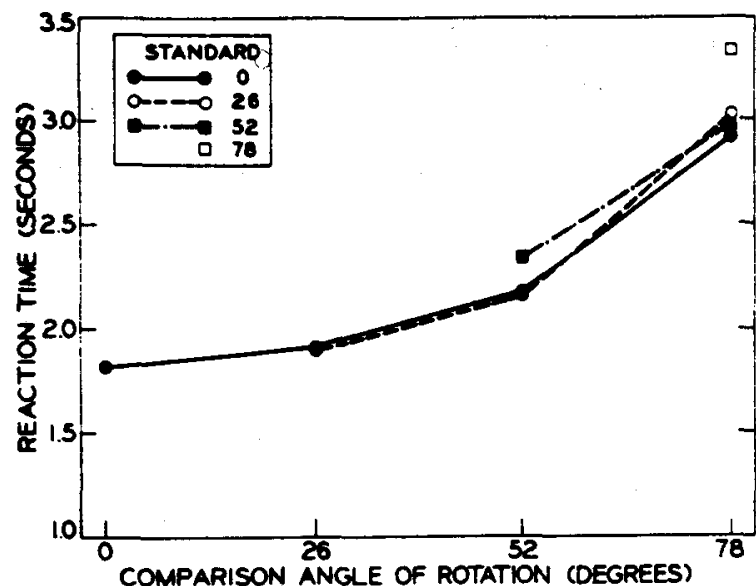

Fig. 5. Mean RTs of correct shape judgments in Experiment III as a function of the angle of rotation of the standard and comparison figures. (In this task, Ss were required to respond same or different with respect to objective shape.)

\section{EXPERIMENT III}

\section{Method}

\section{Subjects}

The five Ss received credit towards a requirement for an introductory course.

\section{Procedure}

The Ss were tested on successive days for five sessions. The practice trials were the same as those used in Experiment II. For expository convenience, one member of each pair is designated as standard and the other as comparison. The standard figures were presented at one of four rotations: $0,26,52$, and $78 \mathrm{deg}$. The standard figure was always the least rotated figure. The comparison figure was presented at the same rotation as the standard or rotated further in depth, if possible, with the constraint that it was presented at one of the four rotations, 0 , 26,52 , or $78 \mathrm{deg}$. Therefore, there were a total of 10 rotation combinations: $0-0,0-26,0-52,0-78,26-26,26-52,26-78,52-52$, 52-78, and 78-78. Each of four figure combinations was presented at each rotation combination. Also, the position of the standard figures was counterbalanced, giving a total of 80 trials per day. The Ss were told to respond "same" or "different" with respect to objective shape. The left and right figures were rotated clockwise and counterclockwise, respectively, since the direction of rotation did not matter in Experiment II.

\section{Results}

Figure 5 and Table 2 present the mean RTs and errors as a function of the orientation of the two figures. The RTs and errors are directly related to the absolute rotation of the two figures and cannot be described adequately as a function of the relative rotation differences. For example, both figures presented in the FPP give a mean response time of $1.823 \mathrm{sec}$, whereas both figures oriented at $78 \mathrm{deg}$ give a mean latency of $3.336 \mathrm{sec}$.

\section{EXPERIMENT IV}

Experiment IV was designed to determine whether the increase in RT reflects the time necessary to determine slant information, Ss taking longer to determine the slant of an object as it is rotated in depth. Once slant is determined, perception of shape could then be independent of absolute rotation, as predicted by the invariance hypothesis. To test this, Experiment III was replicated, asking for same or different judgments with respect to slant rather than shape. Asking Ss for slant judgments eliminates the need to determine true shape, since slant can be determined from information (depth cues) that is independent of shape. If slant judgment times increase with increases in rotation in the same way as shape judgments, it could be concluded that processing time to determine slant is the critical factor in the task. In this case, the invariance hypothesis could provide an acceptable account of the temporal course of shape judgments in the task.

\section{Method}

\section{Subjects}

Five Ss were recruited for credit in an introductory psychology course.

\section{Procedure}

The procedure of Experiment IV was an exact replication of Experiment III, except that Ss were instructed to make objective slant judgments instead of objective shape judgments. They were told that the figures could be the same or different in shape, but that they were to respond same or different with respect to slant. To keep half the trials the same and half different, additional same-slant trials had to be added in the trial sequence. Four of the 10 rotation conditions in Experiment III were the same slant. To achieve an equal number of same and different trials, the conditions were randomized in three blocks of 96 trials each. Seventy-two trials were presented each experimental day.

\section{Results}

The mean RTs in Fig. 6 indicate that the time to determine the slant of a figure is not directly related to

Table 2

Mean Percentage Errors in Experiment III as a Function of the Angle of Rotation of the Standard and Comparison Figures*

\begin{tabular}{|c|c|c|c|c|}
\hline \multirow{2}{*}{$\begin{array}{l}\text { Standard } \\
\text { Angle of } \\
\text { Rotation } \\
\text { (Degrees) }\end{array}$} & Comparison & Angle & Rotation & (Degrees) \\
\hline & 0 & 26 & 52 & 78 \\
\hline $\begin{array}{r}0 \\
26 \\
52 \\
78\end{array}$ & 1.2 & $\begin{array}{l}4.2 \\
3.6\end{array}$ & $\begin{array}{l}3.6 \\
5.3 \\
5.9\end{array}$ & $\begin{array}{r}8.0 \\
5.9 \\
13.0 \\
17.1\end{array}$ \\
\hline
\end{tabular}

"Ss were required to respond "same" or "different" with respect to objective shape. 
increases in the absolute rotation of the figures. The mean percentage errors presented in Table 3 support the RT data. Consistent with the general principle that RT for "different" responses decreases as the differences increase, "different" RTs should decrease with increases in differences in slant. Figure 6 shows this to be the case. However, to determine if two slants are the same, the $\mathrm{S}$ must determine if two figures are the same with respect to slant. The RTs for same responses are $1.637,1.937$, 1.778 , and $1.634 \mathrm{sec}$ for absolute slants of $0,26,52$, and $78 \mathrm{deg}$ of rotation, respectively. Therefore, the increase in RT with increases in rotation in judging true shape cannot be attributed to the time it takes to perceive slant.

\section{EXPERIMENT V}

In the framework of the invariance hypothesis, the remaining possibility is the time it takes to determine the projected shape. In the present task, as in most shape judgments, the projected images of the two figures (circle and ellipse) become more similar as the figures are rotated in depth. By analogy with the task of distinguishing between pairs of frontal-parallel shapes, e.g., pairs of ellipses, it seems likely that discrimination of projective shape would become more difficult as the figures were rotated in depth. This analysis suggests that the operation of determining the projected shape may be the critical process that is dependent upon rotation. If this is the case, it should be just as difficult to judge frontal-parallel projections as it is to judge the projections from figures rotated in depth. In other words, RT should be dependent upon the similarity of the two-dimensional projections presented in the FPP in the same way that it is dependent upon rotation of figures in depth. If this is true, the results can be explained by the fact that figures are more difficult to

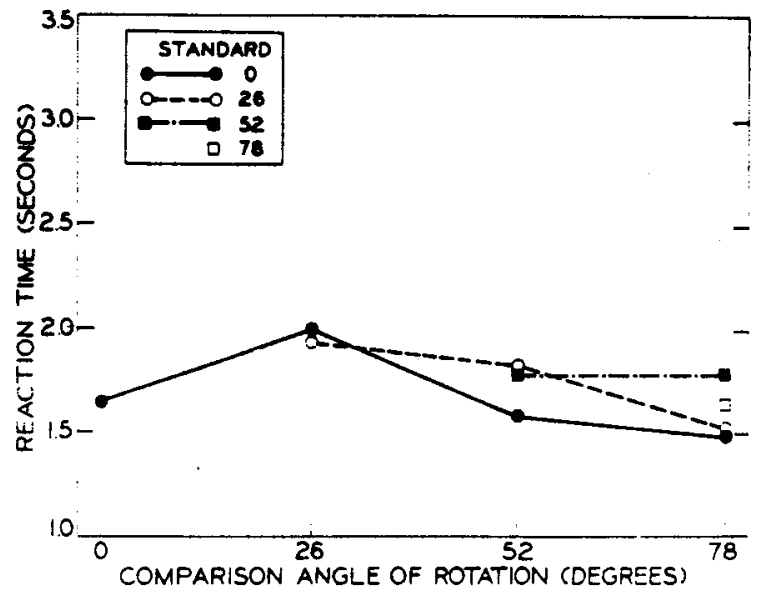

Fig. 6. Mean RTs of correct judgments in Experiment IV as a function of the angle of rotation of the standard and comparison figures. (In this task, Ss were required to respond same or different with respect to objective slant.)
Table 3

Mean Percentage Errors in Experiment IV as a Function of the Angle of Rotation of the Standard and Comparison Figures*

\begin{tabular}{|c|c|c|c|c|}
\hline \multirow{2}{*}{$\begin{array}{l}\text { Standard } \\
\text { Angle of } \\
\text { Rotation } \\
\text { (Degrees) }\end{array}$} & Comparison & Angle & of Rotation & (Degrees) \\
\hline & 0 & 26 & 52 & 78 \\
\hline $\begin{array}{r}0 \\
26 \\
52 \\
78\end{array}$ & 0.0 & $\begin{array}{l}2.0 \\
8.6\end{array}$ & $\begin{array}{l}0.0 \\
9.8 \\
5.3\end{array}$ & $\begin{array}{l}0.8 \\
0.0 \\
9.1 \\
1.6\end{array}$ \\
\hline
\end{tabular}

*Ss were required to respond "same" or "different" with respect to objective slant.

perceive when rotated in depth because their two-dimensional projections become more similar. To test this, Experiment III was replicated, but trials were included that contained figures in the FPP that gave the same projected images as the-figures rotated in depth. If there are no differences between these two types of trials (rotated and frontal-parallel), then increasing RT with increases in orientation is due to the corresponding increases in similarity of the two-dimensional projections.

Since the two retinal projections of the figures differ, it was necessary to take the projection of the figures at a point halfway between the two eyes. The extreme slant of $78 \mathrm{deg}$ had to be eliminated in the present experiment since the projections of the circle and ellipse were practically equal (cf. Table 4). This fact contradicts the invariance hypothesis, which states that the projected image shape and slant are sufficient for reliable shape judgments. The $S$ should not have been able to perform the shape judgment task at the 78-deg rotation according to the invariance hypothesis. This follows from the fact that the projected shapes of the circle and ellipse would be essentially indistinguishable at the $78-\mathrm{deg}$ rotation. In the present study, figures were presented at $65 \mathrm{deg}$ instead of $78 \mathrm{deg}$ so that all the figures representing projected shape in the frontal-parallel plane could have different and, therefore, discriminable projections for the circle and ellipse.

\section{Method}

\section{Subjects}

Four Ss were recruited from an introductory course and were given credit for participation in the experiment. The fifth $S$ was paid $\$ 10$ for participation.

\section{Procedure}

Experiment III was replicated, with the exception that a 65-deg rotation was substituted for the 78-deg rotation. Projected-shape trial types were added to provide a test of the hypothesis that the increase in RT with increases in angle of rotation is due to increased difficulty in discriminating projected shape. Projected shapes were determined for the figures presented at 0.26 .52 . and $65 \mathrm{deg}$. Of course. the projections of the figures at 0 deg are equal to the objective shape of the 
Table 4

Projections of the Horizontal Axes of the Circle and Ellipse at Each Level of Absolute Rotation of the Figure

\begin{tabular}{|c|c|c|c|c|}
\hline \multirow[b]{2}{*}{$\begin{array}{l}\text { Rotation } \\
\text { (Degrees) }\end{array}$} & \multicolumn{2}{|r|}{ Circle } & \multicolumn{2}{|r|}{ Ellipse } \\
\hline & $\mathrm{mm}$ & $\begin{array}{c}\text { Visual } \\
\text { Angle }\end{array}$ & $\mathrm{mm}$ & $\begin{array}{c}\text { Visual } \\
\text { Angle }\end{array}$ \\
\hline $\begin{array}{r}0 \\
26 \\
52 \\
65 \\
78\end{array}$ & $\begin{array}{c}51 \\
43 \\
26 \\
17 \\
5.5\end{array}$ & $\begin{array}{l}4 \mathrm{deg} \\
3 \mathrm{deg} 24 \mathrm{~min} \\
2 \mathrm{deg} 2 \mathrm{~min} \\
1 \mathrm{deg} 20 \mathrm{~min} \\
0 \mathrm{deg} 26 \mathrm{~min}\end{array}$ & $\begin{array}{l}43 \\
36 \\
22 \\
13.6 \\
4.7\end{array}$ & $\begin{array}{l}3 \mathrm{deg} 24 \mathrm{~min} \\
2 \mathrm{deg} 50 \mathrm{~min} \\
1 \mathrm{deg} 44 \mathrm{~min} \\
1 \mathrm{deg} 4 \mathrm{~min} \\
0 \mathrm{deg} 22 \mathrm{~min}\end{array}$ \\
\hline
\end{tabular}

figures. Table 4 presents the lengths and the projected visual angles of the horizontal axes of the circle and ellipse at each angle of rotation. These pairs of projections were presented in the frontal-parallel plane. The projection trials correspond to the trials in which the figures are presented at the same slant. The figures were either the same or different in objective shape, depending on whether the projections were from the same figure or from the two different figures. Therefore, half of the projected-shape trials were the same in objective shape. The projected-shape trials will be compared directly to their analogous trials that involve a rotation in depth. For example, when the figures are rotated at $65 \mathrm{deg}$, the large RT could be due to the fact that the two-dimensional projections are difficult to discriminate. This hypothesis is tested when these projections are presented in the frontal-parallel plane. If the hypothesis is correct, RT should not differ under the two conditions. If RT is larger under the rotated than under the projected conditions, the increase in similarity in the projections with increases in rotation cannot account for the RT increases in the earlier experiments.

There were 432 trials across the 4 experimental days. Two hundred and ninety-eight of the trials were exactly the same as in Experiment III, and 144 of the trials involved the projected shape conditions ( 4 projected slants by 4 figure combinations by 9 replications). One hundred and eight trials were presented per day. As in Experiment III, Ss were instructed to respond same or different on the basis of objective shape and to be as accurate as possible.

\section{Results}

The results indicate that the similarity in projected images. with increases in rotation cannot account for the basic finding. Figure 7 shows that RT increased from 1.948 to $3.280 \mathrm{sec}$ with increases in rotation of the figures presented at the same slant in depth. However, RTs to the figures presented in the FPP increased only slightly $(1.976$ to $2.133 \mathrm{sec})$ as the projected images became more similar. The mean percentage errors presented in Table 5 are positively correlated with the RTs, as in the other experiments. The larger increase in RT at 52 deg FPP projection can be accounted for by the fact that the two of the figures that required a same response differed slightly because one of the figures had a slightly. pointed edge. The Ss reported that this was confusing and slowed them down on both same and different trials. The results then support the notion that discrimination of projections becomes slightly more difficult as the projections become more similar. However, these results cannot account for the large increases in RT with increases in degree of rotation in the experiments.

\section{GENERAL DISCUSSION}

The results contradict the assumption that shape perception can be understood by analyzing the processes involved in perceiving projected shape and slant of the figure. The experiments have shown that the time to encode shape perceptually is dependent on the degree of rotation of the figure. In contrast, the time to encode slant information and projected image information cannot account for the increase with increases in degree of rotation. In the terminology of the invariance hypothesis, the time to obtain information about projected shape and slant does not depend on degree of rotation, whereas the time to perceive objective shape is critically dependent on degree of rotation.

These results strongly indicate that the invariance hypothesis does nul provide a useful model of shape judgment processes. One could argue that, within the framework of the invariance hypothesis, increases in RT reflect increases in the number of times the geometrical algorithm is carried out. However, this assumption could not handle the results found in Experiment II. In that study, Ss knew that the figures were always presented at the same slant and Experiment IV showed that Ss could make same slant judgments easily at all degrees of rotation. Therefore, a decision could have been made on the basis of projected shape information alone. However, RTs increased with increases in absolute orientation. The fact that Ss discriminate these projections rapidly if they are presented in the FPP (Experiment V) shows that two identical retinal projections are not processed in the same way. Retinal projections from figures rotated in depth are much more difficult to analyze for either true shape or projected shape than are projections presented in the frontal-parallel plane. The circle and ellipse gave essentially equal projected shapes at the 78.deg rotation.

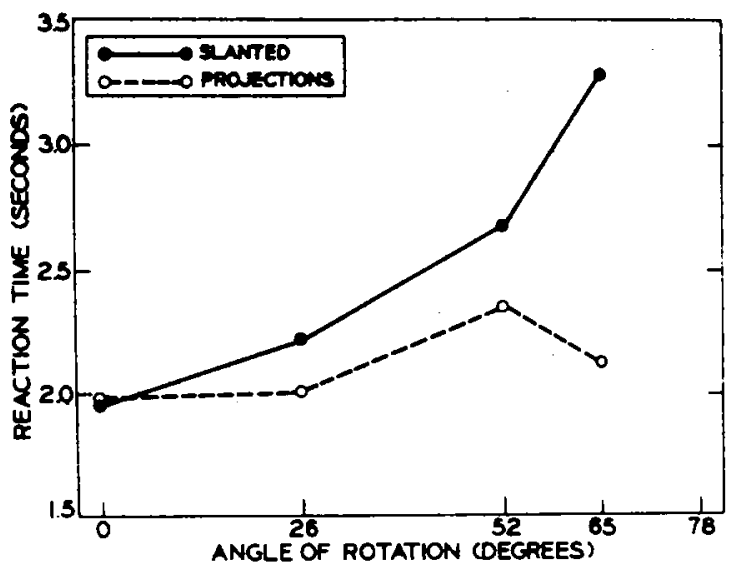

Fig. 7. Mean RTs of correct judgments in Experiment $\mathrm{V}$ as a function of the angle of rotation of the figures. (In the projected condition, the projections of the rotated figures were presented in the FPP.) 
However, Ss were still able to discriminate the figures at this rotation. This should not be possible according to the invariance hypothesis. These observations, taken together with the results of the present research, demonstrate that the invariance hypothesis does not provide a sufficient account of shape constancy. Although projected shape judgments in the FPP and slant judgments can be made easily, perception of true shape involves encoding a figure-ground relationship by a process that does not rely exclusively on the discrete values of projected shape and slant.

It could be argued that $S$ does not have access to the slant and projected shape information that is incorporated in the invariant algorithm. Accordingly, the slant and projected shape judgments in Experiments IV and $\mathrm{V}$ cannot be used as tests of the invariance hypothesis. This formulation of the theory makes the theory essentially untestable and irrelevant to most experimental findings. More importantly, previous results taken as support of the theory would now be irrelevant. For example, demonstrating a positive relationship between slant and true shape judgments (Kaiser, 1967; Winnick \& Rogoff, 1965) would be as inapplicable for the theory as the present experiments. In summary, if the present experiments do not disprove the theory, they at least put it in a position of being untestable and, therefore, unacceptable as a theory of shape perception. Even so, we cannot expect that the invariance hypothesis will be discarded until another theory takes its place.

Massaro ${ }^{1}$ has presented a perceptual encoding model of the shape judgment process that can describe the effects of rotation on reaction time. The central assumption of the model is that perceptual encoding time is a direct function of the time it takes to fuse the two retinal inputs into one picture. Accordingly, perceptual encoding time will increase with increases in fusion time. Fusion time is a direct function of the difficulty of the fusion process. Fusion becomes more difficult and eventually impossible with increases in distance from the horopter.

When the eyes are fixated at a given point in space, points on the horopter stimulate corresponding points of the two retinas. In the present task, the horopter ${ }^{2}$ can be approximated by a circle that passes through the centers of curvatures of the two eyeballs and the point of fixation. Fusion is easier at the horopter and becomes more difficult and eventually impossible with increases in distance from the horopter. Fusion usually does not occur at distances of $6-10 \mathrm{~min}$ or greater from the horopter without noticeable blurring or double images (Ogle, 1964).

In the shape judgment task, an object rotated in depth outside of the fusion area would produce blurring and double images which would make shape judgments more difficult. In this case. additional vergence movements would be necessary to produce a new fixation point which defines a new horopter for fusion. When an $\mathrm{O}$ is
Table 5

Mean Percentage Errors in Experiment $V$ as a Function of the Angle of Rotation of the Figures*

\begin{tabular}{|c|c|c|c|c|}
\hline \multirow[b]{2}{*}{ Condition } & \multicolumn{4}{|c|}{ Angles of Rotation (Degrees) } \\
\hline & 0 & 26 & 52 & 65 \\
\hline Slanted & 1.1 & 1.6 & 3.7 & 18.2 \\
\hline Projections & 1.1 & 0.6 & 5.3 & 6.7 \\
\hline
\end{tabular}

presented with a simple two-dimensional object rotated in depth, vergence eye movements are necessary to determine the shape of the figure. The vergence movement occurs to fixate the eyes at the appropriate point in space. It is necessary to fixate at the depth of the figure so that the images stimulate corresponding points of the retinas, making fusion possible. Consider the case in which the figure is rotated in depth. If the $O$ verges at the axis of rotation of the figure, more of the figure will be off of the horopter as it is rotated in depth and, therefore, less of the figure can be fused.

This encoding model can also account for the finding that the time to make slant judgments does not depend on degree of rotation. In the slant judgment task, the 0 does not have to resolve the true shape of the figure. Since the figures are flat, there should be sufficient information within any small region of the figure for accurate slant judgments at all degrees of rotation. Given that only local information around the horopter is necessary for slant judgments, perceptual encoding time for slant should not increase with increases in degree of rotation of the figure. Accordingly, the encoding model is also consistent with the finding that the time for slant judgments remains constant across degree of rotation.

Finally, Massaro ${ }^{1}$ has presented evidence that the critical independent variable in the shape judgment task is not the angle of rotation of the figure, but rather the distance between the nearest and farthest points of the test figures. Employing test figures which had narrower horizontal axes than those used in the present experiments decreased the effect of angle of rotation. Reaction times to the narrow test figures did not increase sharply with increases in angle of rotation. These results, contrasted with the results with the wide figures used here shows that the extension in depth of the figure is the critical variable that affects perceptual encoding time. The differences found between the narrow and wide test figures cannot be accounted for by either the invariance hypothesis or mental rotation. To date, then, the perceptual encoding model provides the best description of the time it takes to determine the shape of a figure when it is rotated in depth.

\section{REFERENCES}

Beck, J., \& Gibson, J. J. The relation of apparent shape to apparent slant in the perception of objects. Journal of Experimental Psychology, 1955. 50. 125-133.

Lpstein. W.. \& Park, J. N. Shape constancy: functional 
relationships and theoretical formulations. Psychological Bulletin, 1963, 60, 265-288

Kaiser, P. K. Perceived shape and its dependency on perceived slant. Journal of Experimental Psychology, 1967, 75, 345-353.

Koffka, K. Principles of gestalt psychology. New York: Harcourt Brace, 1935.

Leibowitz, H., Mitchell. E., \& Angrist, N. Exposure duration in the perception of shape. Science, 1954, 120, 400.

Ogle, K. Binocular vision. New York: Hafner, 1964.

Shepard, R. N., \& Metzler, J. Mental rotation of three-dimensional objects. Science, 1971, 171, 701-703.

Shipley, T., \& Rawlings, S. C. The nonius horopter. 1. History and theory. Vision Research, 1970, 10, 1225-1262.

Smith, A. H. Gradients of outline convergence and distortion as stimuli for slant. Canadian Journal of Psychology, 1956, 10, 211-218.

Sternberg, S. The discovery of processing stages: Extensions of Donder's method. Acta Psychologica, 1969, 30, 276-315.

Warren, R. M., \& Warren, R. P. Helmholtz on perception: Its physiology and development. New York: Wiley, 1968

Winnick. W. A., \& Rogoff, I. Role of apparent slant in shape judgments. Journal of Experimental Psychology, 1965, 69, 554-563.

Winnick. W. A., \& Rosen, B. E. Shape-slant relations under reduction conditions. Perception \& Psychophysics, 1966, 1, 157-160.

\section{NOTES}

1. Massaro, D. W. Shape perception: Inductive inference as perception encoding. Submitted for publication.

2. Shipley and Rawlings (1970) present a good historical and theoretical discussion of the horopter.

(Received for publication September 13, 1972; revision received January $8,1973$. ) 